\title{
El principio de autonomía e independencia de las cooperativas desde una perspectiva histórica y doctrinal ${ }^{1}$
}

\author{
Alejandro Martínez Charterina \\ Catedrático Emérito de la Universidad de Deusto
}

Sumario: El cuarto principio cooperativo y sus antecedentes. Análisis del principio. Relación del principio con los valores cooperativos. Inquietudes y planteamientos recientes relativos a la autonomía e independencia de las cooperativas. Bibliografía.

Resumen: El principio de autonomía e independencia de las cooperativas encuentra su antecedente en el de neutralidad política y religiosa. Constituye una respuesta a las relaciones que las cooperativas sostienen tanto con los gobiernos en determinados países, como con distintas organizaciones privadas en busca de financiación externa, para preservar su autonomía y su forma de gestión democrática.

Palabras clave: Cooperativas, identidad cooperativa, principios cooperativos.

Abstract: The principle of autonomy and independence of cooperatives can be traced back to that of political and religious neutrality. It is a response to the relationships that cooperatives have both with governments in certain countries and with different private organisations in search of external financing, in order to preserve their autonomy and their form of democratic management.

Keywords: Cooperatives, cooperative identity, cooperative principles.

1 Texto basado en la ponencia del mismo título presentada en el Congreso de la Asociación Internacional de Derecho Cooperativo y la Academia Vasca de Derecho sobre «El principio de autonomía e independencia de las cooperativas», en Bilbao, el 21 de noviembre de 2018. 


\section{El cuarto principio cooperativo y sus antecedentes}

Este cuarto principio de autonomía e independencia de las cooperativas que nos ocupa es uno de los dos nuevos principios, el otro es el séptimo de interés por la comunidad, en la Declaración de la $\mathrm{ACl}$ sobre la identidad cooperativa de 1995.

El enunciado del principio en el texto de la Declaración es el siguiente:

«Las cooperativas son organizaciones autónomas de autoayuda, gestionadas por sus socios. Si firman acuerdos con otras organizaciones, incluidos los gobiernos, o si consiguen capital de fuentes externas, lo hacen en términos que aseguren el control democrático por parte de sus socios y mantengan su autonomía cooperativa»².

De la lectura del texto se desprende con inmediatez que las cooperativas en determinados países precisan algún tipo de ayuda de los gobiernos, y en otros necesitan capital externo a la propia cooperativa para afianzar su competitividad. Se mueven entre el Estado, con referencia a los países en vías de desarrollo, y el mercado, en los países desarrollados, y, siendo entidades autónomas gestionadas democráticamente por sus socios, deben asegurar su autonomía y control democrático.

Como venimos diciendo se trata de un nuevo principio, y responde a la demanda que hace el Informe Böök, preparatorio de la revisión de los principios cooperativos en el Congreso Centenario de la Alianza de 1995. En este informe se recomienda, teniendo en cuenta la experiencia del tiempo anterior y la interrelación de los principios con los valores, un nuevo principio que asegure «...un grado apropiado de independencia y autonomía de las cooperativas», y añade que el mismo puede combinarse con el principio sobre la formación del capital ${ }^{3}$.

Sin embargo no podemos dejar de considerar la vinculación que existe entre el principio y la exigencia de neutralidad política y religiosa que operó como principio cooperativo desde los orígenes de la cooperativa de Rochdale hasta la reforma de los principios por la Alianza Cooperativa Internacional en su XV Congreso de París de 1937, en que dejó de ser un principio necesario y pasó a ser simplemente recomendable, para desaparecer completamente en la reforma del XXIII Con-

2 I.C.A.: Declaración de la Alianza Cooperativa Internacional sobre la Identidad Cooperativa, (1996), p. 19.

3 Sven Ake Böök, Co-operative values in a changing world, (1992), p.17. 
greso de Viena de 1966: «La idea en la que se sustenta el actual cuarto principio cooperativo se encontraba ya presente en los orígenes del Movimiento Cooperativo y, aunque no figuraba todavía de forma explícita en los primeros Estatutos de los Pioneros de Rochdale, se incluye en su posterior modificación de 1850»4.

En efecto, la neutralidad política y religiosa se contempla entre los principios de Rochdale en el estudio que realiza Paul Lambert en base a los Estatutos de la Rochdale Society of Equitable Pioneers de 1844, las enmiendas de los mismos de 1845, y diversos acuerdos de asambleas posteriores hasta llegar a los Estatutos de $1855^{5}$.

En el mismo sentido se expresa desde su constitución en 1895 la Alianza Cooperativa Internacional, que introduce esta neutralidad en el artículo 2. ${ }^{\circ}$ de sus Estatutos: "La Alianza no se ocupa ni de religión ni de política. La Cooperación es un terreno neutral en el que personas que profesen las opiniones y creencias más diversas pueden encontrarse y actuar en común. Para mantener la neutralidad de la que depende la unidad del movimiento cooperativo, toda persona y toda asociación que se adhiere a la Alianza reconoce que la Cooperación se basta a sí misma y no debe servir de instrumento a ningún partido» ${ }^{6}$.

Del mismo modo el informe Böök considera que a pesar de que el principio de neutralidad política y religiosa llegó a desaparecer de forma explícita en el Congreso de Viena, como hemos mencionado anteriormente, implícitamente se ha venido considerando la autosuficiencia plenamente vinculada a la democracia cooperativa. Y por ello el informe propone se lleve a cabo una formulación de este tipo: «Las cooperativas necesitan un grado de autonomía adecuado en sus asuntos internos en orden a su desarrollo efectivo, y deben buscar en consecuencia una independencia apropiada en sus relaciones con gobiernos, autoridades estatales y partidos políticos». ${ }^{7}$

La preocupación de la Alianza Cooperativa Internacional por la autonomía de las cooperativas y el ejercicio de la democracia en las mismas se ha puesto de manifiesto de forma constante a través de sus Congresos.

4 Laura Gómez Urquijo y Alejandro Martínez Charterina, "Origen y alcance del principio de autonomía e independencia de las Cooperativas», (1999), p. 26. En este trabajo se relaciona el principio de autonomía e independencia con la neutralidad política y religiosa, y se examinan las dificultades para la independencia de las cooperativas en los países en vías de desarrollo.

5 Paul Lambert, La doctrina cooperativa, (1970), ps. 57, 86 y 87.

6 Alliance Co-operative Internationale, Deuxième Congrès. Compte Rendu Officiel, (1897), p. 152.

7 Sven Ake Böök, Co-operative values...,o. c.,(1992), p. 232. 
Al mismo tiempo, la Alianza ha tenido en cuenta la importancia y necesidad de la intervención de los gobiernos para el despegue y la buena marcha de las cooperativas en los países en desarrollo.

De este modo considera en el Congreso de Lausana de 1960 la necesidad de apoyo a las cooperativas con carácter general a través de la dotación de infraestructuras adecuadas, ayuda financiera e información, y creando un clima propicio para la buena marcha de estas empresas $^{8}$.

Y en el Congreso de Hamburgo de 1969 se insiste en la importancia que tiene una legislación favorable en los países en desarrollo para la implantación y la buena marcha de las cooperativas ${ }^{9}$.

Siendo así, sin embargo, las experiencias muestran que en ocasiones las cooperativas se han convertido en instrumento oficial de desarrollo en determinadas economías aportando «graves consecuencias al chocar los intereses políticos y económicos del gobierno con los de los socios, no habiendo conseguido encontrar un equilibrio entre ellos» ${ }^{10}$.

Del mismo modo, en sentido contrario, se encuentran muestras de desarrollo cooperativo con ayuda del gobierno, como el caso ejemplar de India y el proyecto de Pandit Nehru, primer ministro desde la independencia del país, que proyectaba la reforma del mismo mediante la cooperación evitando las «desastrosas consecuencias» del control estatal ${ }^{11}$.

Por ello, si bien en ocasiones la Alianza se ha mostrado favorable a la colaboración de los Estados con las cooperativas, también, a partir de un momento ha ido poniendo límites a esa intervención. En el Informe que presentó Alex F. Laidlaw en el XXVII Congreso de la Alianza, de Moscú, en 1980, se considera como uno de los grandes problemas que tendrá que afrontar el cooperativismo ${ }^{12}$. Y para ello propone a los gobiernos «apoyo y ayuda financiera, pero sin dominar, dejando a los cooperativistas que participen en la planificación de la economía nacional en el caso de que las cooperativas sean usadas por el Estado como instrumentos de desarrollo económico» ${ }^{13}$.

8 International Co-operative Alliance, Report of theTwenty-first Congress at Laussane, (1960), p. 171.

9 International Co-operative Alliance, Report of theTwenty-fourth Congress at Hamburg, (1970), p. 286.

10 Laura Gómez Urquijo y Alejandro Martínez Charterina, o.c, (1999), p. 34.

11 J.M. Rana, Forms of Governmet Aid and Co-operative Democracy in South-East Asia, (1973), p. 26.

12 Alex F. Laidlaw, «Las cooperativas en el año 2000», (1981), p. 122.

13 Laura Gómez Urquijo y Alejandro Martínez Charterina, o.c, (1999), p. 35. 
Y es justamente ésta la posición mantenida en la preparación del ajuste de los principios de cara al Congreso centenario de Manchester. Las cooperativas son acreedoras del apoyo que los gobiernos pueden prestarles, especialmente en sus comienzos, tanto con unas estructuras económicas sólidas, como con una legislación particular adecuada, así como con ayuda financiera, apoyo estatal que encuentra sus límites necesarios en cuanto la intervención impide la independencia de la cooperativa en su gestión y control democráticos ${ }^{14}$.

La doctrina se ha alineado con la Alianza advirtiendo de los déficits de democracia en las cooperativas de los países en desarrollo por el control gubernamental. Dante Cracogna señala que «la democracia se halla amenazada por la debilidad o pérdida de autonomía e independencia de las cooperativas frente a los gobiernos u organismos políticos» ${ }^{15}$.

Y Johnston Birchall se refiere a cooperativas creadas por los gobiernos en estos países que deben mantener el control democrático por parte de los socios ${ }^{16}$.

Ahora bien, junto a esa reclamación de autonomía e independencia frente a los Estados, Gobiernos y autoridades, demandada por las experiencias de las cooperativas de Asia y África y también de algunos países de Iberoamérica, relacionadas con los procesos de desarrollo, en las cooperativas en el continente europeo se va a reclamar la independencia cooperativa en los procesos de relación con otras organizaciones no cooperativas para obtener el capital necesario para competir en condiciones adecuadas en un mercado cada vez más grande.

Las cooperativas, en este sentido, ante la necesidad de aumentar su capital en cantidades que los socios no pueden aportar pero que resultan necesarias para el desarrollo competitivo de la actividad, incorporan al socio inversor o capitalista externo que no participa de la actividad de la cooperativa.

El principio de gestión democrática venía exigiendo la aplicación de límites que determinaran el máximo de capital externo a la cooperativa que fuera admisible (en todo caso inferior al 50\%), así como el máximo de votos para los socios externos (por ejemplo, el 25\%) ${ }^{17}$.

14 En este sentido Bruce Thordarson, «From Stockholm to Stockholm: The Lessons of Three Decades of Cooperative Development», (1988), ps.113 ss.

15 Dante Cracogna: «La identidad cooperativa en un mundo cambiante», (1994), p. 92.

16 Johnston Birchall, «Co-operative principles ten years on», (2005), p. 55.

17 Alejandro Martínez Charterina, «El principio de gestión democrática de los socios desde una perspectiva histórica y doctrinal», en Marta Enciso y Eba Gaminde, directoras: Estudio sistemático del principio cooperativo de gestión democrática (2018), p. 27. 


\section{Análisis del principio}

El texto del principio, recogido al comienzo de este trabajo, empieza destacando la esencia de la cooperativa, a la que define como organización autónoma de autoayuda, gestionada por sus socios. Se recoge la idea de Fauquet de que la cooperativa es una asociación de personas que comparten unas necesidades comunes y quieren satisfacerlas a través de una empresa ${ }^{18}$, es decir, la cooperativa organización autónoma de autoayuda, pero además gestionada por sus socios, gestionada democráticamente, según el segundo de los principios cooperativos, como reconoce el texto del principio en su continuación inmediata.

En efecto, los acuerdos posibles que la cooperativa pueda llevar a cabo con cualquier otra organización, privada o pública, así como la obtención de capital de fuentes externas a la cooperativa, no pueden poner en peligro la autonomía de la misma, ya que debe quedar asegurado el control democrático por los socios.

Una vez más se pone de manifiesto la esencia de la identidad de las cooperativas en el texto de este cuarto principio.

Ahora bien, las cooperativas están sujetas a la legislación de los países, y se sienten afectadas por la política económica de los gobiernos de los mismos. Tanto lo uno como lo otro puede resultar beneficioso o perjudicial para ellas.

Del mismo modo las cooperativas compiten en los mercados con el resto de las empresas, y los procesos de globalización conllevan cambios necesarios para mantener la competitividad.

Todo ello conduce a que las cooperativas que entren en contacto con organismos públicos y privados buscando colaboración a través de acuerdos. En esas relaciones tienen que seguir manteniendo su autonomía e independencia, "...la libertad de controlar su propio destino futuro.... ${ }^{19}$.

\section{Relación del principio con los valores cooperativo}

Aunque los valores y los principios que conforman la identidad cooperativa constituyen una unidad y deben considerarse en su conjunto, este principio de autonomía e independencia de las cooperativas nos

18 Georges Fauquet, «Le secteur coopératif», e Oeuvres (1965), ps. 39-40.

19 I.C.A.: Declaración..., o. c., p. 59 
permite profundizar más intensamente en los valores de autoayuda, autorresponsabilidad y democracia.

La autoayuda tiene su fundamento «en la creencia de que todo el mundo puede y debería esforzarse por controlar su propio destino» 20 . A partir de esta consideración la empresa cooperativa se forma por la unión de las personas que deciden compartir esa ayuda transformándola en una ayuda mutua para satisfacer sus necesidades compartidas. Esa decisión promueve la unidad y la independencia en la marcha de la empresa.

La autorresponsabilidad o responsabilidad propia hace que los socios asuman la responsabilidad de la marcha y del devenir de la cooperativa. Y también supone que se sientan «responsables de asegurarse que su cooperativa permanece independiente de otras organizaciones públicas o privadas» ${ }^{21}$.

El valor de la democracia acredita que quienes toman las decisiones en la cooperativa son los socios, cada persona un voto. Evidentemente la democracia cooperativa respalda la autonomía de la misma en sus decisiones frente a cualquier pretensión por parte de otra entidad.

Y estos valores que se tratan de alcanzar actuando conforme indican los principios cooperativos reciben un refuerzo constante del principio de educación y formación, que muestra a los socios de la cooperativa cuál es su esencia y cuál debe ser su conducta, también en la autonomía e independencia debida.

\section{Inquietudes y planteamientos recientes relativos a la autonomía e independencia de las cooperativas}

El «Plan para una década cooperativa» de la Alianza Cooperativa Internacional dedica dos de los cinco temas críticos interrelacionados al cuarto principio cooperativo que nos ocupa, el cuarto «Garantizar marcos jurídicos que apoyen el crecimiento cooperativo», y el quinto "Obtener capital fiable para las cooperativas al mismo tiempo que se garantice el control por parte de los socios»22.

20 I.C.A.: Declaración..., o. c., p. 37.

21 I.C.A.: Declaración..., o. c., p. 37.

22 Alianza Cooperativa Internacional, Plan para una década cooperativa (2013), ps. 26 y 32. 
En base a la Resolución 56/114 de las Naciones Unidas ${ }^{23}$ y la Recomendación 193 de la Organización Internacional del Trabajo24, se pide tanto una legislación como unas políticas que faciliten el desarrollo de las cooperativas teniendo en consideración no sólo que son empresas en el mercado como las que no son cooperativas, sino también los beneficios económicos y sociales que aportan ${ }^{25}$.

Por su parte "obtener capital fiable para las cooperativas» significa por un lado que las cooperativas precisan más capital que el que sus socios y los bancos cooperativos pueden aportar, y, por otro, se trata de una «proposición financiera que ofrezca un rendimiento, pero sin destruir la identidad cooperativa» ${ }^{26}$. Eso hace que el capital sea fiable.

Por parte de la doctrina, Birchall se refiere a la dificultad para el cumplimiento de este cuarto principio en la medida en que no se está consiguiendo que los gobiernos de países en vías de desarrollo abandonen el control de los sectores tradicionales de las cooperativas, por una parte, y por otra, en cuanto la búsqueda de capital por cooperativas de mayor dimensión, y en los procesos de expansión en los mercados mundiales, entre las que considera a cooperativas de la Corporación Mondragón y grandes cooperativas agrícolas, ha llevado a experimentar en ocasiones una pérdida de control por parte de los socios ${ }^{27}$.

Lo cierto es que con la crisis han aumentado las desigualdades de renta y riqueza, la globalización se ha acelerado y domina el modelo de empresa capitalista, y en este contexto se trata de poner en valor el potencial de las cooperativas para mejorar el nivel de vida de las personas pobres y reducir la desigualdad ${ }^{28}$.

Ante lo que venimos considerando es preciso, desde mi punto de vista, profundizar en los principios cooperativos quinto y sexto.

En efecto, el principio de educación, verdadera regla de oro del cooperativismo, debe operar de forma constante haciendo que los so-

23 Resolución 56/114 de las Naciones Unidas adoptada por la Asamblea General el 19 de diciembre de 2001. Puede verse en http://un.org/esa/socdev/social/papers/coop_ dres.pdf

24 Recomendación 193 sobre la promoción de las cooperativas, adoptada por la Organización Internacional del Trabajo el 20 de junio de 2002. Puede verse en http:// www.ilo.org/dys/normlex/es/f?p=NORMLEXPUB:12100:0::NO::P12100_ILO_CODE:R193

25 Puede verse Alianza Cooperativa Internacional, Notas de orientación para los principios cooperativos, (2015), ps. 51 ss.

26 Alianza Cooperativa Internacional, Plan para una década..., o. c., (2013), p. 33.

27 Johnston Birchall, o. c., (2005), p 56.

28 En este sentido Alianza Cooperativa Internacional, Notas de orientación..., o. c., (2015), p. 50. 
cios de la cooperativa sean conscientes de lo que la cooperativa representa para ellos y de los que ellos representan para la cooperativa.

Es a través de la educación y de la formación, que predica el principio, como los socios conocen su papel en cada momento, se preparan para su actividad y toman conciencia de lo que significan la autonomía e independencia en la salvaguarda de la realización de su proyecto.

El sexto principio, por su parte, se estableció con esta categoría en el Congreso de Viena de 1966 justamente para preparar una respuesta al reto del mercado que se percibía desde bastante tiempo antes. Un mercado que crecía de la mano de la presencia de empresas de mayor dimensión con las que era preciso competir a base de cooperación entre cooperativas. A falta de una mayor dimensión la unión de las cooperativas entre sí habría de ser un buen recurso.

\section{Bibliografía}

Alianza CoOperativa INTERnACIONAL: Notas de orientación para los principios cooperativos, 2015. En www.aciamericas.coop/IMG/pdf/guiadance_notes_ es.pdf

Alianza Cooperativa Internacional: Plan para una década cooperative. Enero 2013. En www.aciamericas.coop/IMG/pdf/ica_blueprint_es.pdf

Alliance Co-Operative InTernationale: Deuxième Congrès. Tenu au Musée social, 5, rue Las-Cases, à Paris du 28 au 31 Octobre 1896. Compte Rendu Officiel, ACl, Paris, 1897.

BIRCHALL, Johnston: «Co-operative principles ten years on», en Review of International Co-operation, Vol. 98, N. ${ }^{\circ} 2$ / 2005. I.C.A., Geneva, ps. 45-63.

Böök, SvenAke: Co-operative values in a changing world (Report to the ICA Congress, Tokio, October 1992), International Co-operative Alliance, Geneva, 1992.

CRACOGNA, Dante: "La identidad cooperativa en un mundo cambiante», en Anuario de Estudios Cooperativos 1993, Universidad de Deusto, Bilbao, 1994, ps. 87-96.

FAuquet, Georges: "Le secteur coopératif», en Oeuvres, Publications de L'INSTITUT des ETUES CoOpÉRATIVES, PARIS, 1965, PS. 21-127.

Gómez URquiJo, Laura y Martínez CharTerina, Alejandro: «Origen y alcance del principio de autonomía e independencia de las cooperativas», en Boletín de la Asociación Internacional de Derecho Cooperativo, n. ${ }^{\circ} 33$, Universidad de Deusto, Bilbao, 1999, ps. 25-40.

International Co-Operative Alliance: Declaración de la Alianza Cooperativa Internacional sobre la Identidad Cooperativa. Los principios cooperativos, Consejo Superior de Cooperativas de Euskadi, Vitoria-Gasteiz, 1996.

International Co-Operative Alliance: Report of theTwenty-First Congress at Laussane, $10^{\text {th }}$ to $13^{\text {th }}$ October 1960, ICA, London, 1960. 
International Co-Operative Alliance: Report of theTwenty-fourth Congress at Hamburg, $1^{\text {st }}$ to $4^{\text {th }}$ September 1969, ICA, London, 1970.

LAIDLAW, Alex F.: "Las cooperativas en el año 2000», en Tribuna Cooperativa, nums. 44-45, Centro Nacional de Educación Cooperativa, Zaragoza, 1981, ps. 11-125.

LAMBERT, Paul: La doctrina cooperativa, 3. ${ }^{a}$ ed. Intercoop, Buenos Aires, 1970.

Martínez ChARTERINA, Alejandro: «El principio de gestión democrática de los socios desde una perspectiva histórica y doctrinal», en ENCISO, Marta y GAMINDE, Eba, directoras: Estudio sistemático del principio cooperativo de gestión democrática, Dykinson, Madrid, 2018, ps. 21-28.

RanA, J.M.:Forms of Governmet Aid and Co-operative Democracy in SouthEast Asia, ICA Regional Office \& Education Centre for South-East Asia, New Delhi, 1973.

THORDARSON, Bruce: «From Stockholm to Stockholm: The Lessons of Three Decades of Co-operative Development», en International Co-operative Alliance, XXIX Congress. Stockholm July 1988. Agenda \& Reports, ICA, Geneva, 1988, ps. 109-131. 